\title{
Thermochemical Nonequilibrium Modeling of Electronically Excited Molecular Oxygen
}

\author{
Jae Gang Kim* and Iain D. Boyd ${ }^{\dagger}$ \\ University of Michigan, Ann Arbor, MI 48109, USA
}

\begin{abstract}
The thermochemical nonequilibrium of the three lowest lying electronic states of molecular oxygen, $\mathbf{O}_{2}\left(X^{3} \Sigma_{g}^{-}, a^{1} \Delta_{g}, b^{1} \Sigma_{g}^{+}\right)$, through interactions with argon is studied in the present work. The multi-body potential energy surfaces of $\mathrm{O}_{2}+\mathrm{Ar}$ are evaluated from the semiclassical RKR potential of $\mathrm{O}_{2}$ in each electronic state. The rovibrational states and energies of each electronic state are calculated by the quantum mechanical method based on the present inter-nuclear potential of $\mathrm{O}_{2}$. Then, the complete sets of the rovibrational stateto-state transition rates of $\mathrm{O}_{2}+\mathrm{Ar}$ are calculated by the quasi-classical trajectory method including the quasi-bound states. The system of master equations constructed by the present state-to-state transition rates are solved to analyze the thermochemical nonequilibrium of $\mathrm{O}_{2}+\mathrm{Ar}$ in various heat bath conditions. From these studies, it is concluded that the vibrational relaxation and coupled chemical reactions of each electronic state needs to be treated as a separate nonequilibrium process, and rotational nonequilibrium needs to be considered at translational temperatures above 10,000 K.
\end{abstract}

\section{Introduction}

$\mathrm{T}^{\mathrm{N}}$ recent years, the hypersonic cruise vehicle technologies of the United States were developed through Iscramjet flight programs including X-51A and X-43A. These flight experiences suggested that there is still a significant lack of basic understanding of the aerothermodynamics around hypersonic vehicles and the flow properties through scramjet engines. In typical flight conditions, the cruise speed is about $2-3 \mathrm{~km} / \mathrm{sec}$ at $15-$ $20 \mathrm{~km}$ altitude. In these flight conditions, the equilibrium temperature immediately behind the shock wave increases to about $5,000 \mathrm{~K}$. Such equilibrium temperature is a mild case compared with the temperatures of hypersonic re-entry capsules. In this mild temperature case, there exists an unusual nonequilibrium thermophysics phenomenon, which is not found in Earth re-entry cases. That is the thermochemical nonequilibrium of atmospheric $\mathrm{O}_{2}$. In typical re-entry speeds above $5-6 \mathrm{~km} / \mathrm{sec}$, the $\mathrm{O}_{2}$ is quickly dissociated. Hence, in the aerothermodynamic analysis of re-entry capsules, the thermochemical nonequilibrium of $\mathrm{O}_{2}$ is treated as less important than those of the other atmospheric gas species. However, in the flight conditions of the hypersonic cruise vehicles, the nonequilibrium dissociation of $\mathrm{O}_{2}$ occurs more slowly than those of Earth re-entry flight conditions. Then the thermochemical nonequilibrium of $\mathrm{O}_{2}$ affects the aerothermodynamics behind the shock wave, the surface chemistry and heat transfer of cruise vehicles, and the inlet flow conditions of the engine. In this respect, the thermochemical nonequilibrium of $\mathrm{O}_{2}$ should be treated as important in aerothermodynamic analysis of hypersonic cruise vehicles.

Towards this end, the thermochemical nonequilibrium of $\mathrm{O}_{2}$ is studied in the present work. In describing the rovibrational nonequilibrium and coupled chemical reactions by high-fidelity models, the state-to-state transition rates of $\mathrm{O}_{2}\left(X^{3} \Sigma_{g}^{-}, a^{1} \Delta_{g}, b^{1} \Sigma_{g}^{+}\right)+\mathrm{Ar}$ are calculated by the quasi-classical trajectory (QCT) method with semi-empirical potential energy surfaces. Then, the thermochemical nonequilibrium studies of $\mathrm{O}_{2}$ for the $X^{3} \Sigma_{g}^{-}, a^{1} \Delta_{g}$, and $b^{1} \Sigma_{g}^{+}$electronic states are carried out by solving the system of master equations by using these complete sets of rovibrational state-to-state transition rates. In improving the thermochemical nonequilibrium model associated with $\mathrm{O}_{2}$, the rovibrational relaxations and coupled chemical reactions of each electronic state of $\mathrm{O}_{2}$ are treated as separate nonequilibrium processes by using the results of the master equation calculations.

\footnotetext{
*Post-doctoral Research Fellow, Department of Aerospace Engineering, 1320 Beal Avenue, Member of AIAA.

${ }^{\dagger}$ Professor, Department of Aerospace Engineering, 1320 Beal Avenue, AIAA Fellow.
} 
Table 1. Hulburt-Hirschfelder potential parameters of $\mathrm{O}_{2}$.

\begin{tabular}{cccccc}
\hline \hline & $a$ & $b$ & $c$ & $D\left(c m^{-1}\right)$ & $r_{e}(\AA)$ \\
\hline$X^{3} \Sigma_{g}^{-}$ & $2.653645 \mathrm{E}+00$ & $1.670039 \mathrm{E}+00$ & $6.141914 \mathrm{E}-02$ & $4.206738 \mathrm{E}+04$ & $1.207520 \mathrm{E}+00$ \\
$a^{1} \Delta_{g}$ & $2.813479 \mathrm{E}+00$ & $1.295439 \mathrm{E}+00$ & $8.567842 \mathrm{E}-02$ & $3.416184 \mathrm{E}+04$ & $1.215630 \mathrm{E}+00$ \\
$b^{1} \Sigma_{g}^{+}$ & $2.904263 \mathrm{E}+00$ & $1.077063 \mathrm{E}+00$ & $9.737680 \mathrm{E}-02$ & $2.887301 \mathrm{E}+04$ & $1.226880 \mathrm{E}+00$ \\
$c^{1} \Sigma_{u}^{-}$ & $2.881693 \mathrm{E}+00$ & $5.115760 \mathrm{E}-01$ & $2.688602 \mathrm{E}-01$ & $9.013086 \mathrm{E}+03$ & $1.517400 \mathrm{E}+00$ \\
$A^{\prime 3} \Delta_{u}$ & $3.407727 \mathrm{E}+00$ & $3.748394 \mathrm{E}+01$ & $3.176391 \mathrm{E}-03$ & $7.381066 \mathrm{E}+03$ & $1.48000 \mathrm{E}+00$ \\
$A^{3} \Sigma_{u}^{+}$ & $3.369515 \mathrm{E}+00$ & $8.635466 \mathrm{E}-01$ & $3.911953 \mathrm{E}-01$ & $6.665665 \mathrm{E}+03$ & $1.521500 \mathrm{E}+00$ \\
$B^{3} \Sigma_{g}^{-}$ & $2.706716 \mathrm{E}+00$ & $5.939189 \mathrm{E}-01$ & $2.804103 \mathrm{E}-01$ & $8.141212 \mathrm{E}+03$ & $1.604260 \mathrm{E}+00$ \\
\hline \hline
\end{tabular}

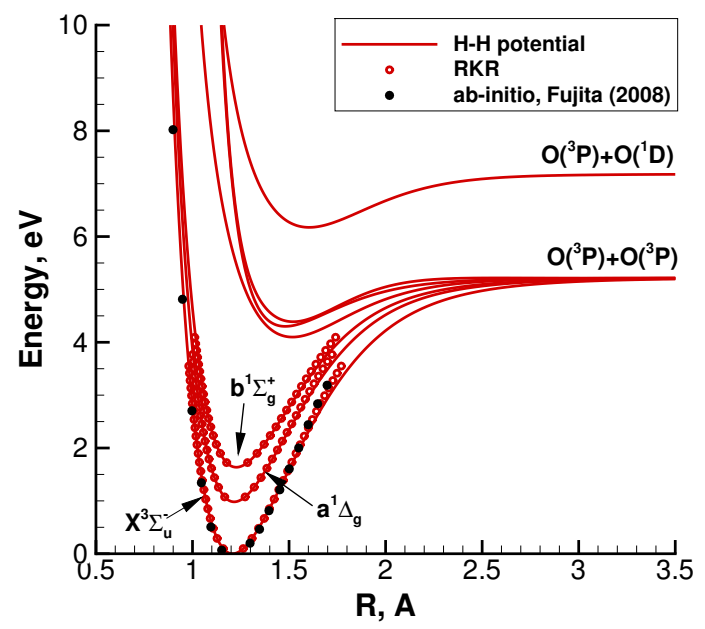

Figure 1. Inter-nuclear potential energy curves of $\mathrm{O}_{2}$.

\section{Molecular structure of $\mathrm{O}_{2}$}

In the present work, the inter-nuclear potential of $\mathrm{O}_{2}$ is represented in an analytical potential function of the Hulburt-Hirschfelder $(\mathrm{H}-\mathrm{H})$ type $^{1}$ as

$$
V_{O_{2}}(r)=D\left[\left(1-e^{-a\left(r-r_{e}\right)}\right)^{2}+c a^{3}\left(r-r_{e}\right)^{3} e^{-2 a\left(r-r_{e}\right)}\left(1+a b\left(r-r_{e}\right)\right)\right] .
$$

The potential parameters $a, b$, and $c$ are determined from a curve-fit of the semi-classically calculated Rydberg, Klein, and Rees (RKR) potential. ${ }^{2}$ In highly-excited states where the RKR potential is unavailable, the $\mathrm{H}-\mathrm{H}$ potential parameters are determined as

$$
\begin{aligned}
a & =\left(k_{e} / 2 D\right)^{\frac{1}{2}}, \\
b & =2-\frac{1}{c}\left[\frac{7}{12}-\frac{1}{a^{2} r_{e}^{2}}\left(\frac{5}{4}+\frac{5 F}{2}+\frac{5 F^{2}}{4}-\frac{G}{12}\right)\right], \\
c & =1-\left(1 / a r_{e}\right)(1+F),
\end{aligned}
$$

where the parameters $\mathrm{F}$ and $\mathrm{G}$ are defined as $F=\alpha_{e} \omega_{e} / 6 B_{e}^{2}$ and $G=8 \omega_{e} x_{e} / B_{e}$, respectively. The potential parameters evaluated in the present work are tabulated in Table 1.

In Fig. 1, inter-nuclear potential energy curves of $\mathrm{O}_{2}$ are presented for each electronic state. $\operatorname{In}_{2}\left(X^{3} \Sigma_{g}^{-}\right)$, the present analytical function of the $\mathrm{H}-\mathrm{H}$ potential agrees with the ab-initio potential energy values calculated by Fujita ${ }^{3}$ and semi-classical RKR potential energy values calculated in the present work. As shown 


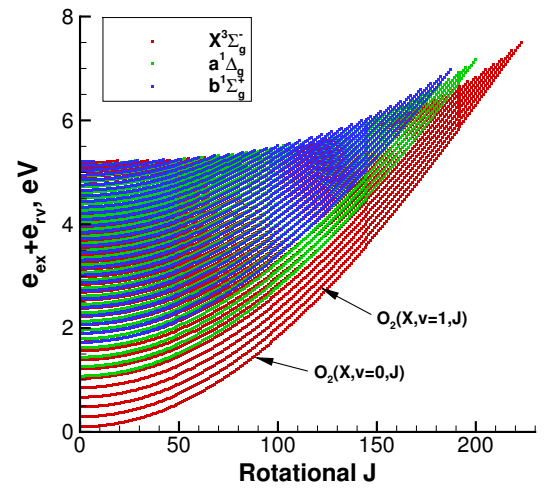

(a) Electronic+rovibratoinal energy

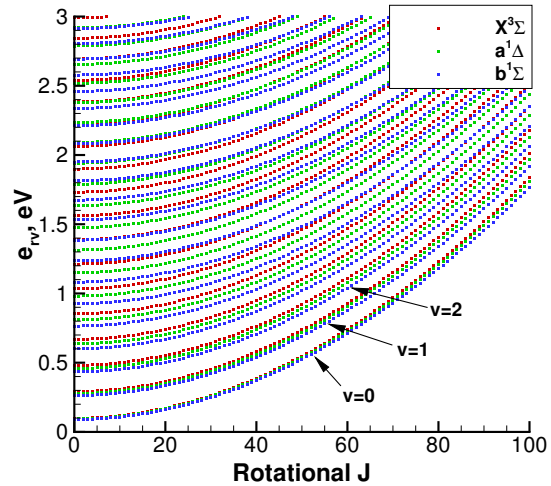

(b) Rovibrational energy

Figure 2. Internal energy of electronic, rovibrational energies of $\mathbf{O}_{2}\left(X^{3} \Sigma_{g}^{-}, a^{1} \Delta_{g}, b^{1} \Sigma_{g}^{+}\right)$.

in Fig. 1, one of the different characteristics of $\mathrm{O}_{2}$ compared with the other atmospheric molecules is that these first three electronic states are closely spaced. The threshold energy between the $X^{3} \Sigma_{g}^{-}-a^{1} \Delta_{g}$ and $a^{1} \Delta_{g}-b^{1} \Sigma_{g}^{+}$transitions is below $1.0 \mathrm{eV}$. This means that electronic excitation occurs easily by electron and heavy-particle collisions. At low atmospheric temperatures, the electronic populations of the $a^{1} \Delta_{g}$ and $b^{1} \Sigma_{g}^{+}$ states are small enough to be negligible. However, immediately behind a shock wave, nonequilibrium excitation by heavy-particle impact occurs rapidly because the heavy-particle translational energy is high enough to excite the electronic states of $\mathrm{O}_{2}$. Such heavy-particle impact excitation phenomena were previously observed in the state-resolved analysis of nitrogen by Kim and Boyd. ${ }^{4}$

In Fig. 2, the evaluated rovibrational energies for the $X^{3} \Sigma_{g}^{-}, a^{1} \Delta_{g}$, and $b^{1} \Sigma_{g}^{+}$states are presented. In the present work, the rovibrational states and energies in each electronic state of $\mathrm{O}_{2}$ are evaluated by the WKB method based on the present $\mathrm{H}-\mathrm{H}$ potential. Then, the total number of rovibrational states of the $X^{3} \Sigma_{g}^{-}, a^{1} \Delta_{g}$, and $b^{1} \Sigma_{g}^{+}$states are 5,117, 3,854, and 3,221, respectively. In figure (a), the summation of the electronic and rovibrational energies are compared for each electronic state. The vibrational energies of $\mathrm{v}=5$ and $\mathrm{v}=9$ in $\mathrm{O}_{2}\left(X^{3} \Sigma_{g}^{-}\right)$are equivalent to the electronic energies of $\mathrm{O}_{2}\left(a^{1} \Delta_{g}\right)$ and $\mathrm{O}_{2}\left(b^{1} \Sigma_{g}^{+}\right)$states, respectively. According to the previous two-temperature (2-T) model, ${ }^{5}$ such vibrational levels are important above $T_{e e v}=5,000 \mathrm{~K}$, and the mole fractions of the $a^{1} \Delta_{g}$ and $b^{1} \Sigma_{g}^{+}$states increase in proportion to $\exp \left(-e_{i} / k T_{e e v}\right)$. Hence, the rovibrational relaxations and coupled chemical reactions of $\mathrm{O}_{2}\left(a^{1} \Delta_{g}\right)$ and $\mathrm{O}_{2}\left(b^{1} \Sigma_{g}^{+}\right)$need to be treated in the thermochemical nonequilibrium analysis of $\mathrm{O}_{2}$ at the scramjet flight conditions. In figure (b), the rovibrational energies of the $X^{3} \Sigma_{g}^{-}, a^{1} \Delta_{g}$, and $b^{1} \Sigma_{g}^{+}$states are compared. The threshold energy from $\mathrm{v}=0$ to $\mathrm{v}=1$ of each electronic state is similar. However, above $\mathrm{v}=3$, the differences of the vibrational threshold energy increase. This means that the vibrational relaxation times for the $X^{3} \Sigma_{g}^{-}, a^{1} \Delta_{g}$, and $b^{1} \Sigma_{g}^{+}$ states are different from each other at high vibrational temperatures where the vibrational number density above $\mathrm{v}=3$ becomes import. In previous aerothermodynamic analysis of $\mathrm{O}_{2}$, the vibrational relaxation of the $X^{3} \Sigma_{g}^{-}, a^{1} \Delta_{g}$, and $b^{1} \Sigma_{g}^{+}$states are treated as a single relaxation phenomenon represented by the lowfidelity model of Millikan-White. ${ }^{6}$ In improving the thermochemical nonequilibrium model associated with electronically excited $\mathrm{O}_{2}$, the vibrational relaxation and coupled chemical reactions of each electronic state needs to be treated as separate nonequilibrium processes with high-fidelity thermochemical nonequilibrium models.

\section{Rovibrational state-to-state transitions of $\mathrm{O}_{2}+\mathrm{Ar}$}

To examine the rovibrational relaxations and coupled chemical reactions of electronically excited $\mathrm{O}_{2}$, the rovibrational state-to-state transition rates of $\mathrm{O}_{2}+\mathrm{Ar}$ are calculated by the QCT method in the present work. Most of the shock-tube measured data were provided for the $\mathrm{O}_{2}+\mathrm{Ar}$ system, and it is more convenient for theoretical analysis because Ar is a noble gas. The goal of the present work is to develop high-fidelity 
Table 2. Buckingham potential parameters of O-Ar. ${ }^{9}$

\begin{tabular}{ccccc}
\hline \hline pair & $\mathrm{A}\left(E_{h}^{-1}\right)$ & $\mathrm{B}\left(a_{0}^{-1}\right)$ & $\mathrm{C}\left(E_{h}^{-1} a_{0}^{6}\right)$ & $r_{m}\left(a_{0}\right)$ \\
\hline $\mathrm{O}-\mathrm{Ar}$ & 209.9357 & 2.0169 & 43.8514 & 6.427 \\
\hline \hline
\end{tabular}

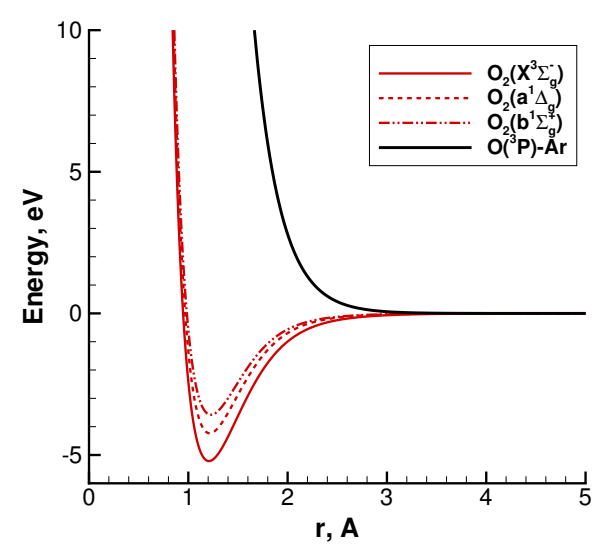

(a) inter-nuclear potential of $\mathrm{O}_{2}$ and $\mathrm{O}-\mathrm{Ar}$

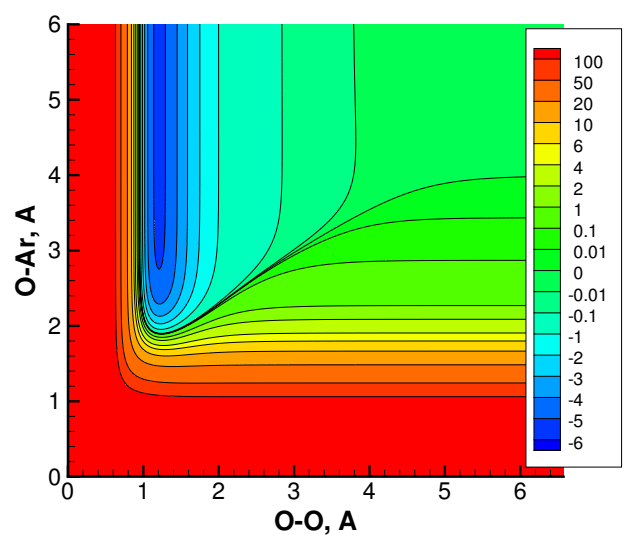

(b) $\mathrm{O}_{2}\left(X^{1} \Sigma_{g}^{-}\right)$-Ar potential energy surface $(\mathrm{eV})$ in $90^{\circ}$ collision

Figure 3. Two- and three-body potential energy surface of O-O-Ar.

thermochemical nonequilibrium models for the $\mathrm{O}_{2}$ system. Hence, the $\mathrm{O}_{2}+\mathrm{Ar}$ system will be extended to the $\mathrm{O}_{2}+\mathrm{O}$ and $\mathrm{O}_{2}+\mathrm{O}_{2}$ systems in the future. However, $\mathrm{O}_{2}+\mathrm{Ar}$ is enough to observe the rovibrational nonequilibrium of electronically excited $\mathrm{O}_{2}$.

In evaluating the complete sets of the transition rates for each electronic excited state, a simple potential energy surface model for $\mathrm{O}_{2}\left(X^{3} \Sigma_{g}^{-}, a^{1} \Delta_{g}, b^{1} \Sigma_{g}^{+}\right)+$Ar is proposed as

$$
V\left(r_{O_{a}-A r}, r_{O_{a}-O_{b}}, r_{O_{b}-A r}\right)=V_{O_{2}}+\Sigma_{i=a, b} V_{O_{i}-A r} .
$$

Argon is a noble gas, and the Van der Waals force between Ar and the other atomic species is so weak as to allow treatment as a purely repulsive potential. This simple type of potential energy surface was adopted in the previous many-body potential work for $\mathrm{O}_{3}+\mathrm{Ar}^{.7,8}$ In the present work, the O-Ar potential is represented using an analytical function of the Buckingham potential ${ }^{7}$ as

$$
V_{O-A r}=A \exp (-B r)-C \exp \left[-\left(\frac{1.28 r_{m}}{r-1.0}\right)^{2}\right] / r^{6} .
$$

The potential parameters A, B, and C are obtained from the work by Kroes and Rettschnick ${ }^{9}$ and tabulated in table 2. Then, the potential energy surfaces of $\mathrm{O}_{2}\left(X^{3} \Sigma_{g}^{-}\right)+\mathrm{Ar}, \mathrm{O}_{2}\left(a^{1} \Delta_{g}\right)+\mathrm{Ar}$, and $\mathrm{O}_{2}\left(b^{1} \Sigma_{g}^{+}\right)+\mathrm{Ar}$ are constructed by using Eqs. 1 to 4.

In Fig. 3, two- and three-body potential energy surfaces of O-O-Ar are presented. In the two-body potentials of figure (a), $\mathrm{O}_{2}\left(X^{3} \Sigma_{g}^{-}\right), \mathrm{O}_{2}\left(a^{1} \Delta_{g}\right), \mathrm{O}_{2}\left(b^{1} \Sigma_{g}^{+}\right)$, and $\mathrm{O}\left({ }^{3} P\right)$-Ar potentials are compared. In $\mathrm{O}\left({ }^{3} P\right)$ Ar interaction, the Van der Waals forces are weak enough to ignore, and the distance where the repulsive force drastically increases is larger than those of the O-O interactions. In the three-body potential energy surface of figure (b), the potential energy surfaces of $\mathrm{O}_{2}\left(X^{3} \Sigma_{g}^{-}\right)+\mathrm{Ar}$ are presented for the $90^{\circ}$ collision trajectory. When Ar approaches $\mathrm{O}_{2}$, the potential energy shape does not change until the distance reaches $4 \AA$. However, the shape is drastically changed from $4 \AA$, and the steepest ascendant potential shape of the repulsive part is generated at $2 \AA$.

In the present work, the rovibrational state-to-state transition rates are calculated by the QCT method based on the present potential energy surfaces of $\mathrm{O}_{2}\left(X^{3} \Sigma_{g}^{-}\right)+\mathrm{Ar}, \mathrm{O}_{2}\left(a^{1} \Delta_{g}\right)+\mathrm{Ar}$, and $\mathrm{O}_{2}\left(b^{1} \Sigma_{g}^{+}\right)+\mathrm{Ar}$. All 


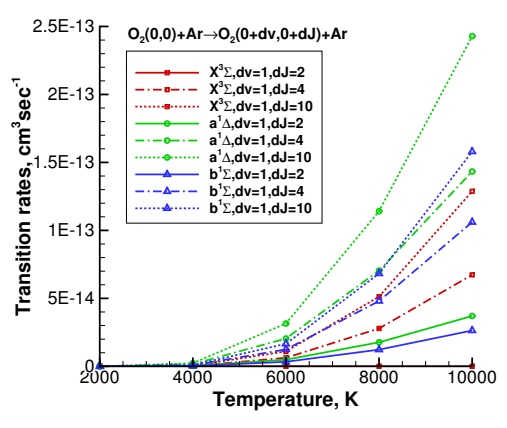

(a)

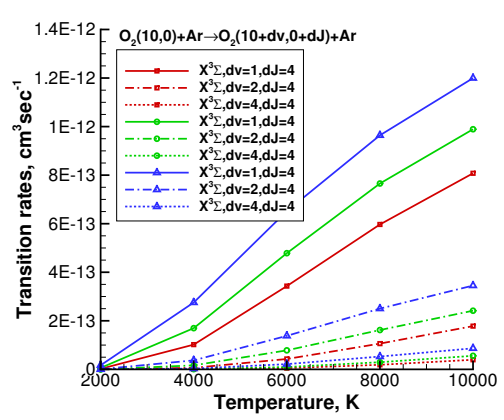

(d)

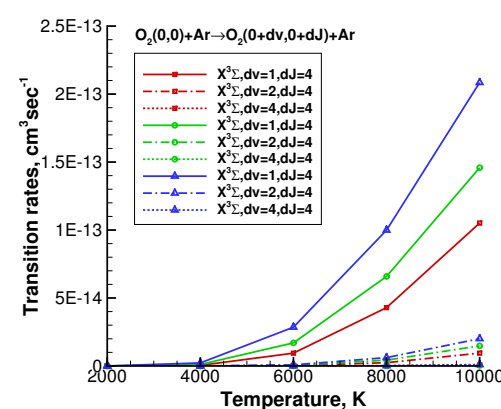

(b)

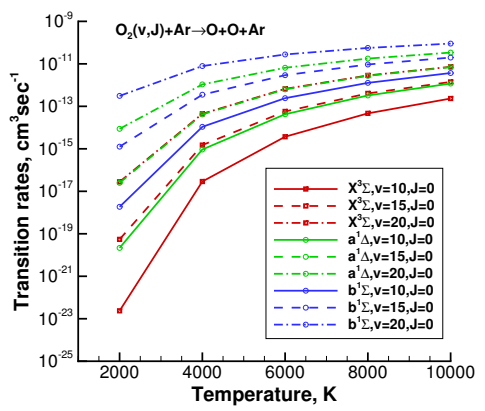

(e)

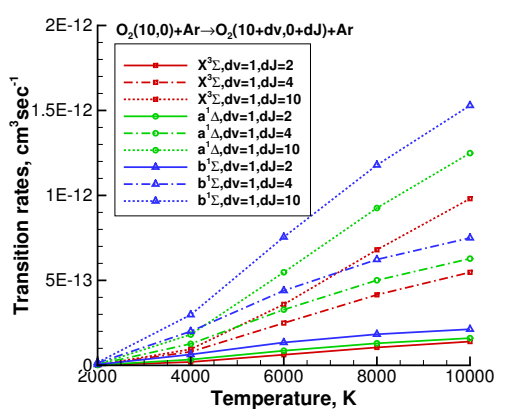

(c)

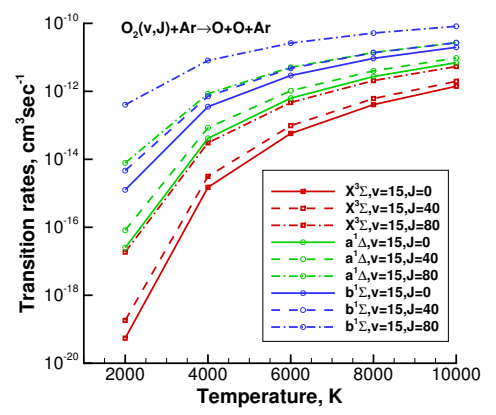

(f)

Figure 4. Comparisons of the bound-bound and bound-free transition rates for $\mathrm{O}_{2}+\mathrm{Ar}$

trajectories are initialized by internal energies that correspond precisely to each of the $5,117,3,854$, and 3,221 rovibrational states of $\mathrm{O}_{2}\left(X^{3} \Sigma_{g}^{-}\right), \mathrm{O}_{2}\left(a^{1} \Delta_{g}\right)$, and $\mathrm{O}_{2}\left(b^{1} \Sigma_{g}^{+}\right)$, respectively. In determining the initial coordinates and the conjugated momenta of $\mathrm{O}_{2}$ given by the present $\mathrm{H}-\mathrm{H}$ potential, a fast Fourier transform method proposed by Eaker ${ }^{10}$ is adopted with some modifications. In the present QCT calculations, stratified sampling is employed to obtain the impact parameter, and all other parameters are integrated by a Monte Carlo method. The final rovibrational states are quantized by the WKB method. In each trajectory calculation, a total of 2000 trajectories are calculated per impact parameter at the batch size of $0.1 \AA$. Further details about the QCT calculations are described in other literature ${ }^{11,12}$

In Fig. 4, the bound-bound and bound-free transition rates for $\mathrm{O}_{2}+\mathrm{Ar}$ are compared for selected transitions. In figures (a) to (d), bound-bound transitions of $\mathrm{O}_{2}(0,0)+\mathrm{Ar}$ and $\mathrm{O}_{2}(10,0)+\mathrm{Ar}$ are compared for the electronically excited states of $\mathrm{O}_{2}$. In the same bound-bound transitions, the rovibrational transition rates of each electronic state $\mathrm{O}_{2}$ are different, and these differences increase in proportion to the temperature and threshold energy for the transitions. Such patterns are also observed in the bound-free transition rates of figures (e) and (f).

\section{Master equation study for bound-bound transitions}

For $\mathrm{O}_{2}\left(X^{3} \Sigma_{g}^{-}, a^{1} \Delta_{g}, b^{1} \Sigma_{g}^{+}\right)+\mathrm{Ar}$, a database of rovibrational state-to-state transition rates are constructed for translational temperatures from $1,000 \mathrm{~K}$ to $20,000 \mathrm{~K}$ including the quasi-bound states of $\mathrm{O}_{2}$. By using the database of transition rates for $\mathrm{O}_{2}+\mathrm{Ar}$, a system of master equations is constructed to model the relaxations of the rotational and vibrational modes. In this section, the bound-free transitions are excluded because the relaxations of the rotational and vibrational modes are mainly produced by the bound-bound transitions. The master equation with this assumption is defined as,

$$
\frac{d n_{i}}{d t}=\sum_{j} K(i, j) n_{A r}\left[\frac{Q_{i}}{Q_{j}} n_{j}-n_{i}\right],
$$



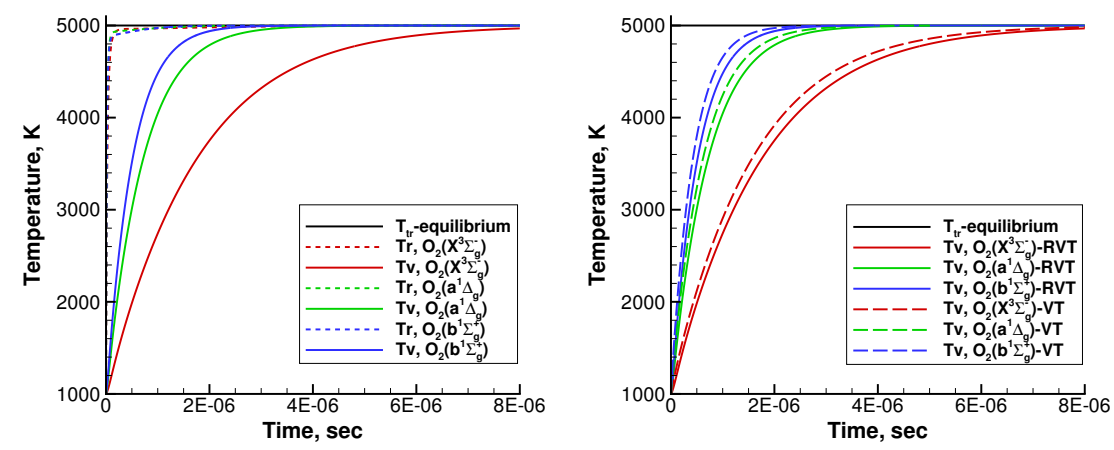

(a) Rotational and vibrational tempera- (b) Vibrational temperatures by RVT and tures by RVT transitions at $\mathrm{T}=5,000 \mathrm{~K} \quad \mathrm{VT}$ transitions at $\mathrm{T}=5,000 \mathrm{~K}$
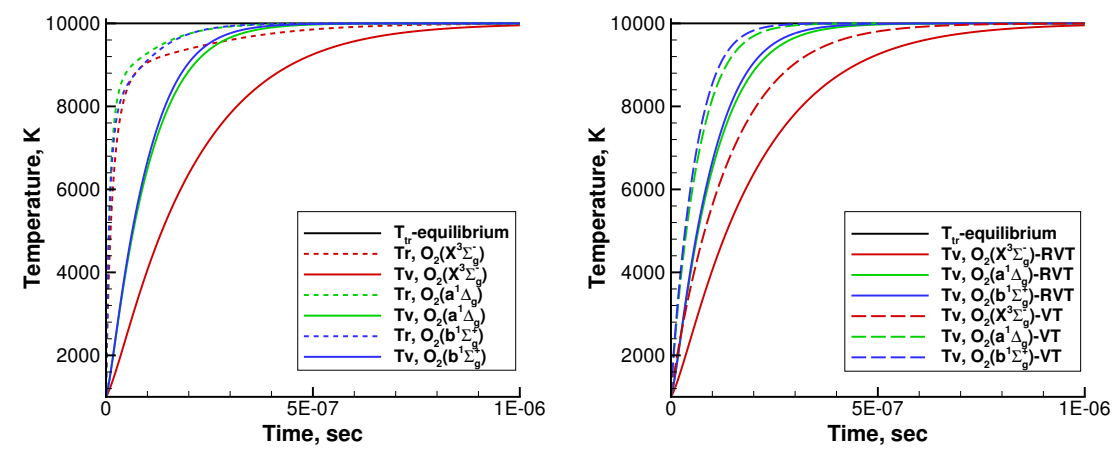

(c) Rotational and vibrational tempera- (d) Vibrational temperatures by RVT and tures by RVT transitions at $\mathrm{T}=10,000 \mathrm{~K} \quad \mathrm{VT}$ transitions at $\mathrm{T}=10,000 \mathrm{~K}$

Figure 5. Rotational and vibrational temperature relaxations of $\mathbf{O}_{2}\left(X^{3} \Sigma_{g}^{-}, a^{1} \Delta_{g}, b^{1} \Sigma_{g}^{+}\right)$.

where $n_{i}$ and $n_{j}$ are the number densities of $i^{t h}$ and $j^{t h}$ rovibrational states of $\mathrm{O}_{2}(v, J)$ and $\mathrm{O}_{2}\left(v^{\prime}, J^{\prime}\right)$, respectively. $n_{A r}$ is the number density of the colliding species. $t$ is time and $K(i, j)$ is the rovibrational state-to-state transition rates of the bound-bound transitions. The rovibrational Boltzmann factor $Q_{i}$ is defined as

$$
Q_{i}=\epsilon g_{e}(2 J+1) g_{s} \exp \left(-\frac{e_{i}}{k T}\right),
$$

where $\epsilon$ is a symmetry factor for $\mathrm{O}_{2} . g_{e}$ and $g_{s}$ are the total degeneracy of the electronic state and nuclear spin degeneracy of $\mathrm{O}_{2}$, respectively. $J$ is the rotational state corresponding to the rovibrational state $i . k$ is the Boltzmann constant and $T$ is the translational temperature. $e_{i}$ is an energy of rovibrational state $i$ in each electronic excited states. The principle of detailed balance between the forward and backward rates is invoked under equilibrium, which leads to

$$
K(i, j) Q_{i}=K(j, i) Q_{j} .
$$

Then, the system of master equations are constructed by Eq. (5) for all 5,117, 3,854, and 3,221 rovibrational states of $\mathrm{O}_{2}\left(X^{3} \Sigma_{g}^{-}, a^{1} \Delta_{g}, b^{1} \Sigma_{g}^{+}\right)$, respectively. In integrating the system of master equations, an implicit time integration method accurate to third order along the diagonal and second order off-diagonal elements is adopted in the present work. The temperature range from 1,000 $\mathrm{K}$ to $20,000 \mathrm{~K}$ is studied for weak and strong nonequilibrium conditions. For all cases, the initial number densities of $\mathrm{Ar}$ and $\mathrm{O}_{2}$ are set to a constant of $5 \times 10^{17} \mathrm{~cm}^{-3}$ and an isothermal condition is applied in these heat bath calculations.

In the present work, energy-equivalent rotational and vibrational temperatures are adopted to characterize the rotational and vibrational modes. These energy-equivalent rotational and vibrational temperatures 


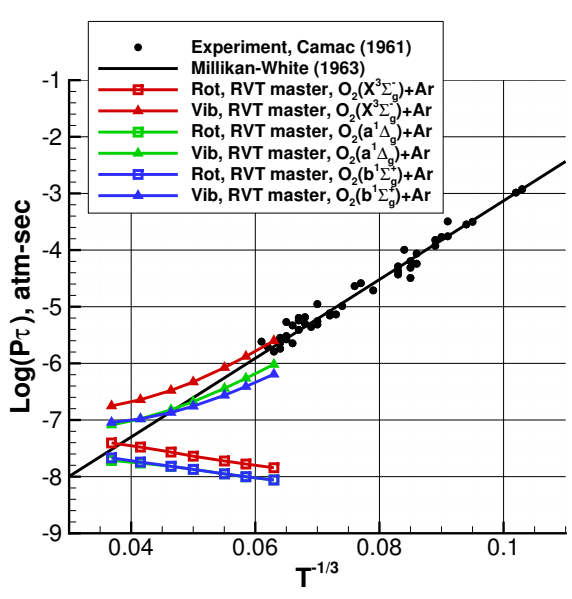

(a) Rotational and vibrational relaxations by RV'T transitions

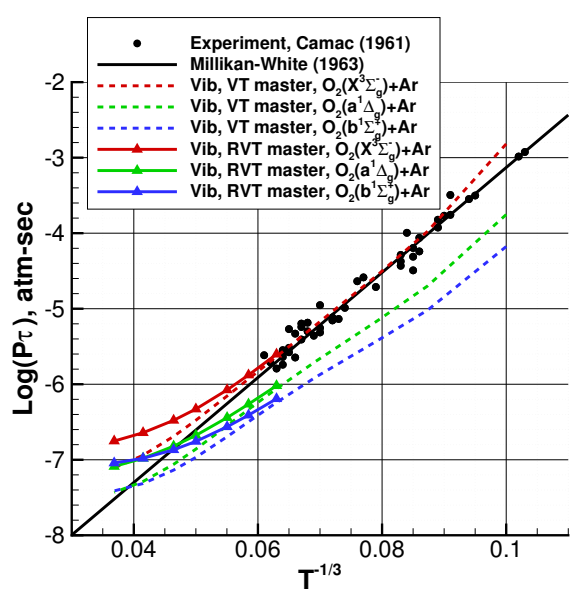

(b) Vibrational relaxations by RVT and VT transitions

Figure 6. Rotational and vibrational relaxations of $\mathrm{O}_{2}\left(X^{3} \Sigma_{g}^{-}, a^{1} \Delta_{g}, b^{1} \Sigma_{g}^{+}\right)$.

can be determined by the average rotational and the vibrational energies, respectively. These average rotational and vibrational energies are defined as

$$
\begin{aligned}
& \widetilde{e}_{r}=\frac{\sum_{i} e_{r}(i) n_{i}}{\sum_{i} n_{i}}, \\
& \widetilde{e}_{v}=\frac{\sum_{i} e_{v}(i) n_{i}}{\sum_{i} n_{i}},
\end{aligned}
$$

respectively. $e_{r}(i)$ and $e_{v}(i)$ are the rotational and vibrational energies corresponding to the rovibrational state $i$, respectively. These rotational and vibrational energies of each electronic state of $\mathrm{O}_{2}\left(X^{3} \Sigma_{g}^{-}, a^{1} \Delta_{g}, b^{1} \Sigma_{g}^{+}\right)$ are evaluated by the quantum mechanical method based on the present $\mathrm{H}-\mathrm{H}$ potential.

In Fig. 5, the rotational and vibrational temperature relaxations by the rotational-vibrational-translational energy (RVT) and vibrational-translational energy (VT) transitions are compared for $\mathrm{O}_{2}\left(X^{3} \Sigma_{g}^{-}, a^{1} \Delta_{g}\right.$, $\left.b^{1} \Sigma_{g}^{+}\right)+$Ar at the equilibrium temperatures of 5,000 K and 10,000 K. For the VT transitions, the system of master equations in each electronic state is constructed by the vibrational state-to-state transition rates of $\mathrm{O}_{2}+\mathrm{Ar}$. The vibrational state-to-state transition rates can be derived from the assumption that the rotational number density distribution is represented by the Boltzmann distribution at the equilibrium temperature $\mathrm{T}$;

$$
K\left(v \rightarrow v^{\prime} ; T\right)=\frac{\sum_{J} Q(J, T) \sum_{J^{\prime}} g_{J^{\prime}} K\left(v, J \rightarrow v^{\prime} J^{\prime} ; T\right)}{\sum_{J} Q(J, T)}
$$

In Figures (a) and (b), the rotational temperatures converge quickly to the equilibrium temperatures for all electronic $X^{3} \Sigma_{g}^{-}, a^{1} \Delta_{g}$, and $b^{1} \Sigma_{g}^{+}$states in the RVT transitions. In comparison of the vibrational temperatures between the RVT and VT transitions of the figures (a) and (b), the differences are small enough to be ignored. However, at the equilibrium temperatures of 10,000 K of figures (c) and (d), the differences of the vibrational temperatures between the RVT and VT transitions are obvious even though the rotational relaxations are much faster than vibrational relaxations. These results show that rotational nonequilibrium needs to be considered at 10,000 $\mathrm{K}$ because the rotational nonequilibrium affects the vibrational relaxation through the rotational to vibrational energy transfer processes. In comparison of the rotational and vibrational relaxations of each of the $X^{3} \Sigma_{g}^{-}, a^{1} \Delta_{g}$, and $b^{1} \Sigma_{g}^{+}$states, the vibrational relaxation of electronic ground $\mathrm{O}_{2}\left(X^{3} \Sigma_{g}^{-}\right)$is much slower than that of the excited states $\left(a^{1} \Delta_{g}, b^{1} \Sigma_{g}^{+}\right)$at temperatures of $5,000 \mathrm{~K}$ and $10,000 \mathrm{~K}$. The vibrational relaxation of $\mathrm{O}_{2}\left(a^{1} \Delta_{g}\right)$ is slightly faster than that of $\mathrm{O}_{2}\left(b^{1} \Sigma_{g}^{+}\right)$at 5,000 K. However, at $T=10,000 \mathrm{~K}$, the vibrational relaxation of $\mathrm{O}_{2}\left(a^{1} \Delta_{g}\right)$ and $\mathrm{O}_{2}\left(b^{1} \Sigma_{g}^{+}\right)$are almost identical.

In Fig. 6, the characteristic relaxation parameters of $p \tau_{r}$ and $p \tau_{v}$ calculated by the RVT and VT transitions for $\mathrm{O}_{2}\left(X^{3} \Sigma_{g}^{-}, a^{1} \Delta_{g}, b^{1} \Sigma_{g}^{+}\right)+\mathrm{Ar}$ are compared with the shock-tube measured data by Camac ${ }^{13}$ and 


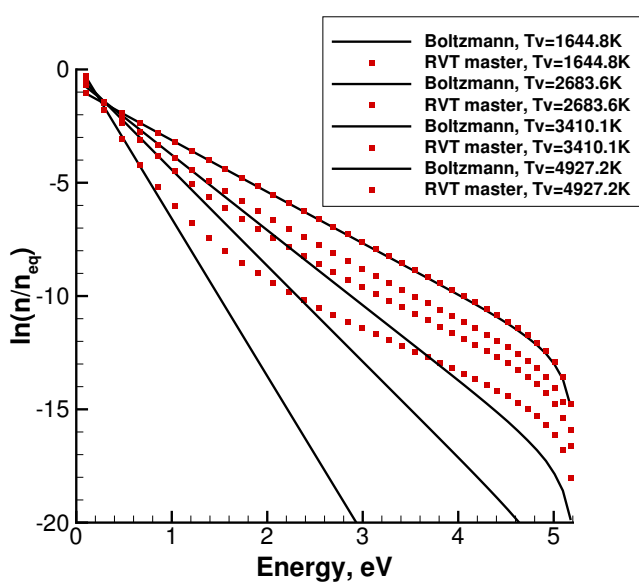

(a) $\mathrm{T}=5,000 \mathrm{~K}$

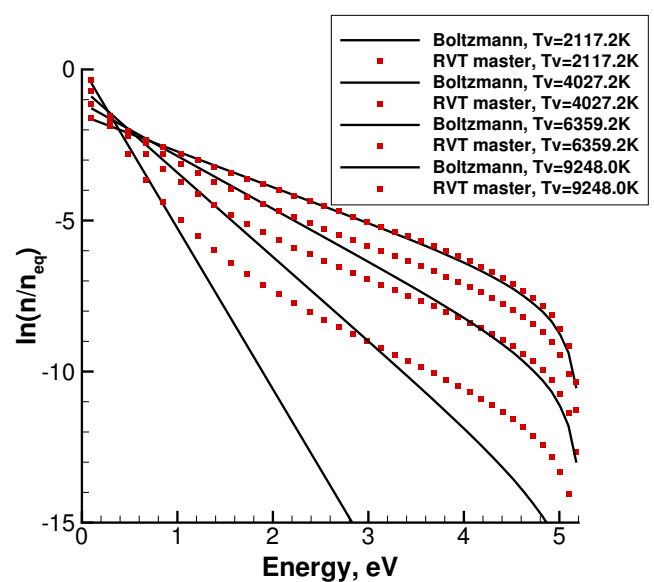

(b) $\mathrm{T}=10,000 \mathrm{~K}$

Figure 7. Vibrational number density distributions of $\mathrm{O}_{2}\left(X^{3} \Sigma_{g}^{-}\right)$.

the empirical equation proposed by Millikan and White. ${ }^{6}$ In the shock-tube experiment by Camac, the vibrational relaxation time of $\mathrm{O}_{2}\left(X^{3} \Sigma_{g}^{-}\right)+\mathrm{Ar}$ was measured through the $\mathrm{O}_{2}$ Schumann-Runge radiation in shock-heated $\mathrm{O}_{2}$-Ar mixtures. As shown in figures (a) and (b), the present vibrational relaxation time calculated by the state-to-state kinetic method with the RVT and VT transitions agrees well with the shock-tube experimental data. In the present work, the relaxation times of the rotational and vibrational modes are defined by using the Landau-Teller form ${ }^{14}$ as

$$
\begin{gathered}
\frac{\partial \widetilde{e}_{r}}{\partial t}=\frac{\widetilde{e}_{r}(T)-\widetilde{e}_{r}}{\tau_{r}}, \\
\frac{\partial \widetilde{e}_{v}}{\partial t}=\frac{\widetilde{e}_{v}(T)-\widetilde{e}_{v}}{\tau_{v}},
\end{gathered}
$$

respectively. $\widetilde{e}_{r}(T)$ and $\widetilde{e}_{v}(T)$ are the average rotational and vibrational energies at equilibrium temperature T. $\tau_{r}$ and $\tau_{v}$ are the rotational and vibrational relaxation times, respectively. By equating Eqs. (11) and (12) with the results of the master equation calculations, the relaxation time can be determined with the $e$-folding collision number method. ${ }^{15}$ By multiplying by the pressure, one obtains the characteristic rotational and vibrational relaxation parameters $p \tau_{r}$ and $p \tau_{v}$, respectively. In figure (a), the rotational relaxation time is faster than that of vibration initially. However, the differences of the relaxation time between the rotational and vibrational modes become small when the temperature increases. At temperatures above 10,000K, the rotational relaxation time of $\mathrm{O}_{2}\left(X^{3} \Sigma_{g}^{-}, a^{1} \Delta_{g}, b^{1} \Sigma_{g}^{+}\right)$is slow enough to be treated as nonequilibrium. In comparisons of the vibrational relaxation of the electronic $X^{3} \Sigma_{g}^{-}, a^{1} \Delta_{g}$, and $b^{1} \Sigma_{g}^{+}$states of $\mathrm{O}_{2}$, the vibrational relaxation between the electronically excited $a^{1} \Delta_{g}$ and $b^{1} \Sigma_{g}^{+}$states is almost identical, and the relaxation of the $X^{3} \Sigma_{g}^{-}$state is much slower than that of the excited states. In typical hypersonic flows, the vibrational nonequilibrium immediately behind a shock wave is treated as one vibrational temperature mode represented by the Landau-Teller equation with the modification factor of vibrational diffusion. ${ }^{16}$ However, the present results show that the vibrational relaxation of each electronic excited state needs to be treated as separate vibrational temperature process. In figure (b), the vibrational relaxation parameters calculated by the RVT and VT transitions are compared. The difference of vibrational relaxation between the RVT and VT transitions is small enough to be ignored at temperatures below 8,000K. However, a large difference of the vibrational relaxation between the RVT and VT transitions is shown above 10,000K where the rotational nonequilibrium affects the vibrational relaxation through the rotational-to-vibrational energy transfer processes.

In Fig. 7, the vibrational number density distributions of $\mathrm{O}_{2}\left(X^{3} \Sigma_{g}^{-}\right)$in each relaxation process at temperatures of $5,000 \mathrm{~K}$ and $10,000 \mathrm{~K}$ are presented. The vibrational number density distributions by the master 
equation calculations are compared with the Boltzmann distributions specified by the nonequilibrium temperatures, and the number density distributions are normalized by their own equilibrium distributions. In figures (a) and (b), strong nonequilibrium distributions are observed at high vibrational energy above $1.0 \mathrm{eV}$. The deviation of the vibrational number density between the master equation calculations and Boltzmann distributions begins from the $\mathrm{v}=5$ state at temperatures of 5,000 $\mathrm{K}$ and $10,000 \mathrm{~K}$. However, in the low vibrational states, where most of the number density exists, the nonequilibrium distributions agree with the Boltzmann distributions.

\section{Master equation study for bound-free transitions}

A system of master equations with nonequilibrium chemical reactions can be constructed with the stateto-state transition rate coefficients in the following form;

$$
\begin{aligned}
\frac{d n_{i}}{d t} & =\sum_{j} K(i, j) n_{A r}\left[\frac{Q_{i}}{Q_{j}} n_{j}-n_{i}\right] \\
& +K(i, c) n_{x}\left[\frac{Q_{i} Q_{t_{O_{2}}}}{Q_{O}^{2} Q_{t_{O}}^{2}} \exp \left(\frac{D_{i}}{k T}\right) n_{O}^{2}-n_{i}\right], \\
\frac{d n_{O}}{d t} & =\sum_{i} K(i, c) n_{A r}\left[n_{i}-\frac{Q_{i} Q_{t_{O_{2}}}}{Q_{O}^{2} Q_{t_{O}}^{2}} \exp \left(\frac{D_{i}}{k T}\right) n_{O}^{2}\right]
\end{aligned}
$$

where $K(i, c)$ are the bound-free transition rates. $D_{i}$ is the dissociation energy of $\mathrm{O}_{2}$ in rovibrational state $i$. $Q_{O}$ and $n_{O}$ are the atomic partition function and species number density of $O\left({ }^{1} P\right)$, respectively. $Q_{t}$ is the translational partition function. The principle of detailed balancing relations for bound-free transitions are

$$
K(i, c) \frac{Q_{i} Q_{t_{O_{2}}}}{Q_{O}^{2} Q_{t_{O}}^{2}} \exp \left(\frac{D_{i}}{k T}\right)=K(c, i) .
$$

By using Eqs. (13) and (15), a system of rovibrational master equations are constructed for each electronic state of $\mathrm{O}_{2}$ including the quasi-bound states and atomic species $\mathrm{O}\left({ }^{1} P, J=0\right.$ to 2$)$. The variation in species number density of $n_{O_{2}}$ and $n_{O}$ are calculated for heating environments for isothermal heat bath conditions. The temperatures range from $1,000 \mathrm{~K}$ to $20,000 \mathrm{~K}$ in the heating cases are studied to provide weak and strong nonequilibrium conditions. For all cases, initial number densities of $\mathrm{Ar}$ and $\mathrm{O}_{2}$ are set to a constant of $1 \times 10^{18} \mathrm{~cm}^{-3}$.

In Fig. 8, the relaxations of the rotational and vibrational temperatures and species number densities of dissociated $\mathrm{O}$ and $\mathrm{O}_{2}$ are presented for the heating cases. In figures (a) and (b), the relaxations of the temperatures and number densities of $\mathrm{O}_{2}\left(X^{3} \Sigma_{g}^{-}, a^{1} \Delta_{g}, b^{1} \Sigma_{g}^{+}\right)$by the RVT transitions are compared at the temperature of $10,000 \mathrm{~K}$. As shown in figures (a) and (b), the rotational and vibrational temperatures quickly converged to the rovibrational quasi-steady state (QSS). During this converging process, chemical reactions occur infrequently. However, most of the chemical reactions occur in the rovibrational QSS period. During this QSS period, the rotational and vibrational temperatures are at almost constant values, and the chemical reactions occur rapidly. In the QSS period, the number density rate of change on the left-hand side in Eq. (13) is much smaller than both the sum of all incoming rates and sum of all outgoing rates on the right-hand side. This phenomenon results in the rotational and vibrational energies maintaining near constant values. In comparisons of the chemical reactions for the electronic $X^{3} \Sigma_{g}^{-}, a^{1} \Delta_{g}$, and $b^{1} \Sigma_{g}^{+}$states, it is observed that the chemical reactions of the excited $\mathrm{O}_{2}\left(a^{1} \Delta_{g}, b^{1} \Sigma_{g}^{+}\right)$are much faster than those of the electronic ground $\mathrm{O}_{2}\left(X^{3} \Sigma_{g}^{-}\right)$in RVT transitions. In figures (c) and (d), the comparisons of the vibrational temperature relaxation and number densities by the RVT and VT transitions are presented. As shown in figures (c) and (d), the vibrational temperature relaxation by the RVT transitions is much slower than that by the VT transitions. However, the nonequilibrium chemical reactions by the RVT transitions are only slightly slower than those by the VT transitions.

In Fig. 9, the rotational and vibrational temperatures and vibrational number density distributions of $\mathrm{O}_{2}\left(X^{3} \Sigma_{g}^{-}\right)+$Ar by the RVT transitions are presented at the temperature of $10,000 \mathrm{~K}$. In Zone 1, the rotational and vibrational temperatures are rapidly converged to the QSS period without chemical reactions. In this process, strong nonequilibrium distributions are observed at $t=1.9 \times 10^{-8} \mathrm{sec}$ and $t=9.9 \times 10^{-8} \mathrm{sec}$. In Zone 1, the deviation of vibrational number density distributions between the RVT master equation 

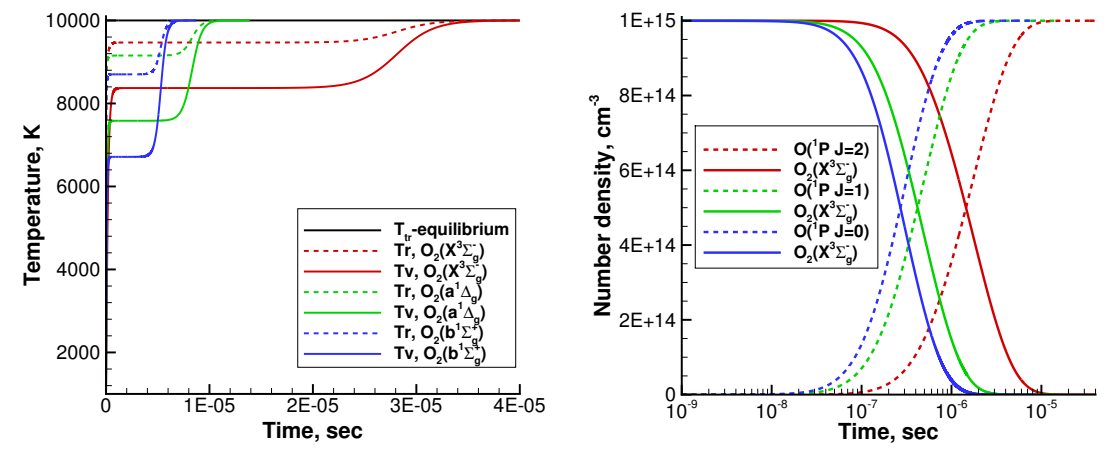

(a) Rotational and vibrational tempera- (b) Vibrational temperatures by RVT and tures by RVT transitions

VT transitions
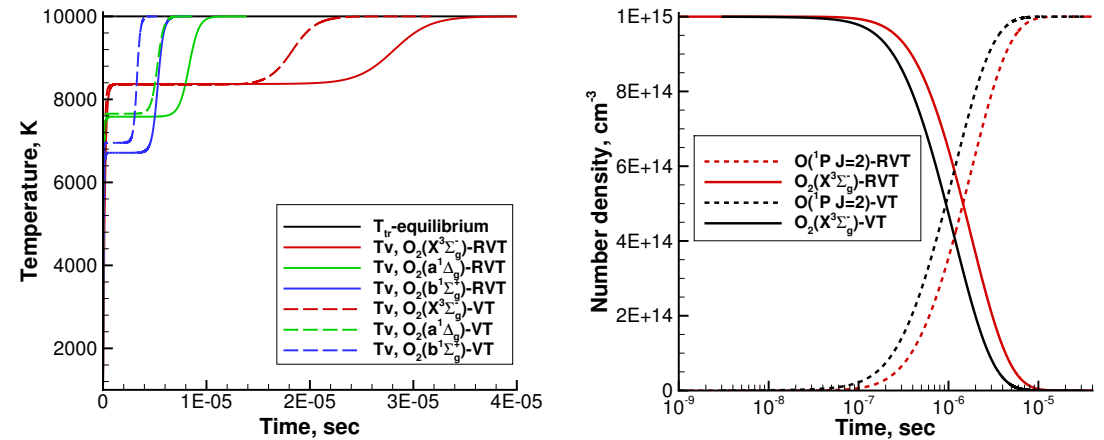

(c) Species number densities by RVT transitions

(d) Species number densities by RVT and VT transitions of $\mathrm{O}_{2}\left(X^{3} \Sigma_{g}^{-}\right)+\mathrm{Ar}$

Figure 8. Rotational and vibrational temperatures and species number densities for $\mathrm{O}_{2}\left(X^{3} \Sigma_{g}^{-}, a^{1} \Delta_{g}, b^{1} \Sigma_{g}^{+}\right)+\mathbf{A r}$ at $\mathrm{T}=10,000 \mathrm{~K}$.

calculations and Boltzmann distributions is large enough to be treated as strong vibrational nonequilibrium. In Zone 2, the vibrational distributions calculated by the RVT master equations have similar distributions represented by the Boltzmann at $t=7.59 \times 10^{-7} \mathrm{sec}$ and $t=9.82 \times 10^{-2} \mathrm{sec}$ except at high vibrational energy. At vibrational energy above $4 \mathrm{eV}$, there still exist deviations between the RVT master equation calculations and Boltzmann distributions because the chemical reactions occur rapidly in this period. In Zone 3, the rotational and vibrational temperatures and number density distributions are fully converged to the equilibrium temperature and Boltzmann distributions specified by the equilibrium temperature of $10,000 \mathrm{~K}$.

In the results of the RVT master equation calculations, it is observed that the nonequilibrium chemical reactions mostly occur in the QSS period, and the number density distributions in the QSS period can be represented by the Boltzmann distributions specified by the nonequilibrium temperature. Then, the dissociation reaction rate coefficients in this QSS period can be derived by solving the set of algebraic equations by using the QSS assumption. The rate of change of $n_{i}$ is defined as

$$
\begin{aligned}
\frac{d n_{i}}{d t} & =\sum_{j} K(j, i) n_{j} n_{A r}-\sum_{j} K(i, j) n_{i} n_{A r} \\
& -K(i, c) n_{i} n_{A r}+K(c, i) n_{O}^{2} n_{A r} .
\end{aligned}
$$

From the assumption of QSS of rotational and vibrational energies, the left-hand side of Eq. (16) can be set to zero. By using normalized populations, $\phi_{i}=n_{i} / n_{i_{E} q}, \phi_{j}=n_{j} / n_{j_{E} q}$, and $\phi_{O}=n_{O} / n_{O_{E} q}$, Eq. (16) 


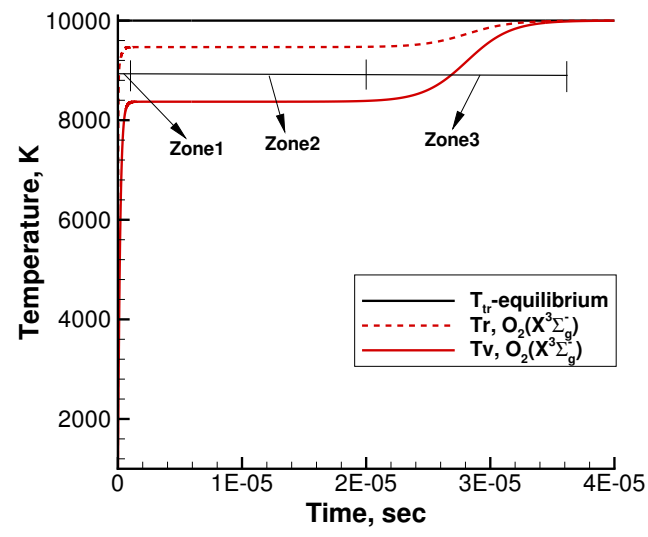

(a) Rotational and vibrational temperatures

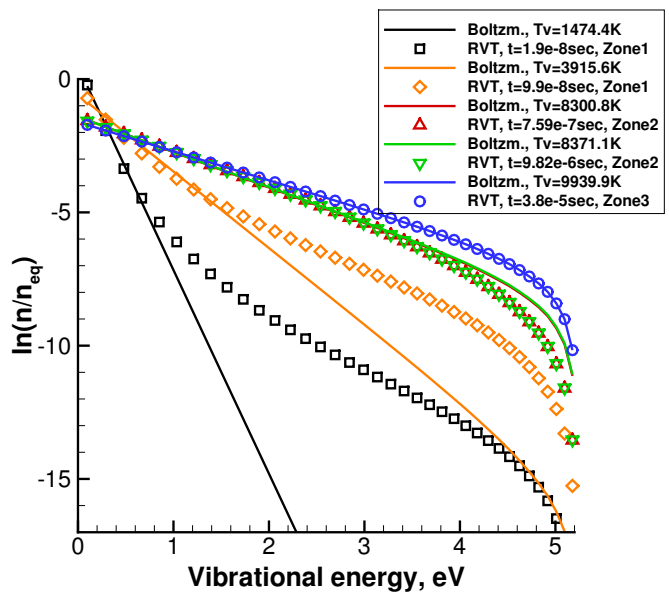

(b) Vibrational number density distributions

Figure 9. Rotational and vibrational temperature relaxtions and vibrational number density distributions of $\mathrm{O}_{2}\left(X^{3} \Sigma_{g}^{-}\right)$by RVT transitions at $10,000 \mathrm{~K}$.

can be rewritten as

$$
\left[\sum_{j} K(i, j)+K(i, c)\right] \phi_{i}-\sum_{j} K(i, j) \phi_{j}=[K(i, c)] \phi_{O}^{2} .
$$

On the other hand, the sum of all $n_{i}$ must equal the given number density of $\mathrm{O}_{2}$; that is,

$$
\sum_{i} n_{i_{E q}} \phi_{i}=n_{O_{2} q} \chi
$$

In Eq. (17), we drop the first equation and substitute using Eq. (18). This approach is based on the intuition that the QSS relation is least likely to be satisfied by the rovibrational ground state of $\mathrm{O}_{2}$. Then Eq. (17) can be rewritten as $5,117 \times 5,117,3,854 \times 3,854$, and $3,221 \times 3,221$ matrices for the electronic $X^{3} \Sigma_{g}^{-}, a^{1} \Delta_{g}$, and $b^{1} \Sigma_{g}^{+}$states of $\mathrm{O}_{2}$, respectively;

$$
\mathbf{M} \vec{\phi}=\vec{C} \phi_{O}^{2}+\vec{D} \chi
$$

The normalized population vector $\vec{\phi}$ can be defined by using homogeneous $\vec{\phi}_{h}$ and particular solutions $\vec{\phi}_{p}$ as

$$
\vec{\phi}=\vec{\phi}_{h}+\vec{\phi}_{p} \phi_{O}^{2}
$$

Then, the homogeneous and particular solutions are

$$
\begin{aligned}
\vec{\phi}_{h} & =\mathbf{M}^{-1} \vec{D} \chi, \\
\vec{\phi}_{p} & =\mathbf{M}^{-1} \vec{C},
\end{aligned}
$$

respectively. By using the normalized populations, the rate of change of $n_{O}$ can be rewritten from Eq. (14) as

$$
\frac{d n_{O}}{d t}=n_{O_{2}} n_{A r} \sum_{i} K(i, c) \frac{Q_{i}}{Q_{m}}\left[\phi_{i}+\phi_{O}^{2}\right],
$$

where $Q_{m}$ is the molecular partition function of $\mathrm{O}_{2}$. By using the homogeneous and particular solutions of Eq. (20), this equation can be derived as follows;

$$
\begin{aligned}
-\frac{d n_{O_{2}}}{d t} & =\frac{d n_{O}}{d t}=n_{O_{2}} n_{A r} \sum_{i}[K(i, c)] \frac{Q_{i}}{Q_{m}} \phi_{i, h} \\
& -n_{O}^{2} n_{A r} \sum_{i}[K(i, c)] \frac{Q_{i}}{Q_{m}} \frac{n_{O_{2_{E q}}}}{n_{O_{E q}}^{2}}\left[1-\phi_{i, p}\right] .
\end{aligned}
$$




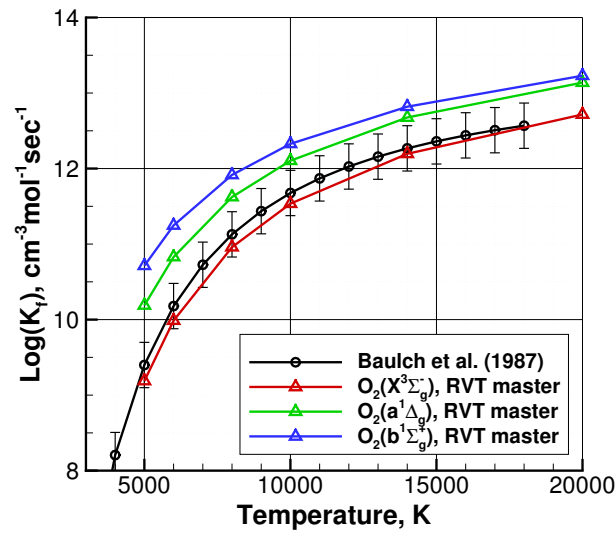

(a) Reaction rates by RVT transitions

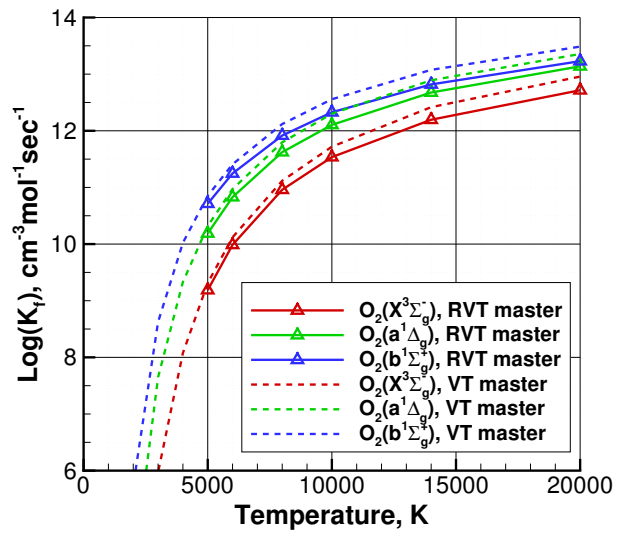

(b) Reaction rates by RVT and VT transitions

Figure 10. Dissociation reaction rates $\mathbf{O}_{2}\left(X^{3} \Sigma_{g}^{-}, a^{1} \Delta_{g}, b^{1} \Sigma_{g}^{+}\right)+\mathbf{A r}$.

Then, we can define the dissociation reaction rate coefficients in the QSS period as

$$
K_{f_{Q S S}}=\sum_{i}[K(i, c)] \frac{Q_{i}}{Q_{m}} \phi_{i, h}
$$

Further details about the derivation of the QSS reaction rates are described in the work by Kim and Boyd. ${ }^{17}$

In Fig. 10, the QSS reaction rates of $\mathrm{O}_{2}\left(X^{3} \Sigma_{g}^{-}, a^{1} \Delta_{g}, b^{1} \Sigma_{g}^{+}\right)+\mathrm{Ar}$ are presented. In figure (a), the reaction rates calculated by the RVT transitions are compared with the experimental data by Baulch et al. ${ }^{18}$ As shown in figure (a), the present QSS reaction rates of $\mathrm{O}_{2}\left(X^{3} \Sigma_{g}^{-}\right)+\mathrm{Ar}$ agree well with the experimental data. The reaction rates of electronically excited $\mathrm{O}_{2}\left(a^{1} \Delta_{g}, b^{1} \Sigma_{g}^{+}\right)$are much faster than the reaction rate of the ground state $\mathrm{O}_{2}\left(X^{3} \Sigma_{g}^{-}\right)$. These results show that the dissociated atomic $\mathrm{O}\left({ }^{1} P\right)$ is efficiently produced from the chemical reaction of electronically excited $\mathrm{O}_{2}\left(a^{1} \Delta_{g}, b^{1} \Sigma_{g}^{+}\right)$. In figure (b), the QSS reaction rates of $\mathrm{O}_{2}\left(X^{3} \Sigma_{g}^{-}, a^{1} \Delta_{g}, b^{1} \Sigma_{g}^{+}\right)+$Ar calculated by the RVT transitions are compared with those by the VT transitions. The reaction rates calculated by the RVT transitions are slightly slower.

In Fig. 11, the rotational and vibrational average energy losses due to dissociation are presented. The average energy loss in the present work is defined as

$$
\widetilde{e}_{x_{l o s s}}=\frac{\sum_{i}\left[K(i, c) n_{A r}\right] e_{x}(i) n_{i_{E q}}\left[\phi_{O}^{2}-\phi_{i}\right]}{\sum_{i}\left[K(i, c) n_{A r}\right] n_{i_{E q}}\left[\phi_{O}^{2}-\phi_{i}\right]}
$$

where $x$ represents the rotational or vibrational mode. This average energy loss is normalized by the dissociation energy of $\mathrm{O}_{2}$. In the figure, it is observed that the normalized vibrational energy loss is about 0.6 at $10,000 \mathrm{~K}$ and this value approaches 0.45 when the temperature increases up to $20,000 \mathrm{~K}$. In the normalized rotational energy loss, the value is about 0.35 at $10,000 \mathrm{~K}$ and approaches 0.45 when the temperature increases. This is because, at low temperatures, most of the dissociation occurs at high-vibrational and low-rotational energy levels. When the temperature increases, the average dissociated energy levels move to lower vibrational and higher rotational energy levels.

\section{Conclusions}

The thermochemical nonequilibrium of the lowest three electronically excited states of $\mathrm{O}_{2}\left(X^{3} \Sigma_{g}^{-}, a^{1} \Delta_{g}\right.$, $\left.b^{1} \Sigma_{g}^{+}\right)+\mathrm{Ar}$ is studied in the present work. The multi-body potential energy surfaces of $\mathrm{O}_{2}+\mathrm{Ar}$ are evaluated from the semi-classical RKR potential of $\mathrm{O}_{2}$ in each electronic state. The rovibrational states and energies of each electronic state are calculated by the quantum mechanical method based on the present inter-nuclear potential of $\mathrm{O}_{2}$. Then, the rovibrational state-to-state transition rates of $\mathrm{O}_{2}\left(X^{3} \Sigma_{g}^{-}, a^{1} \Delta_{g}, b^{1} \Sigma_{g}^{+}\right)+\mathrm{Ar}$ are 


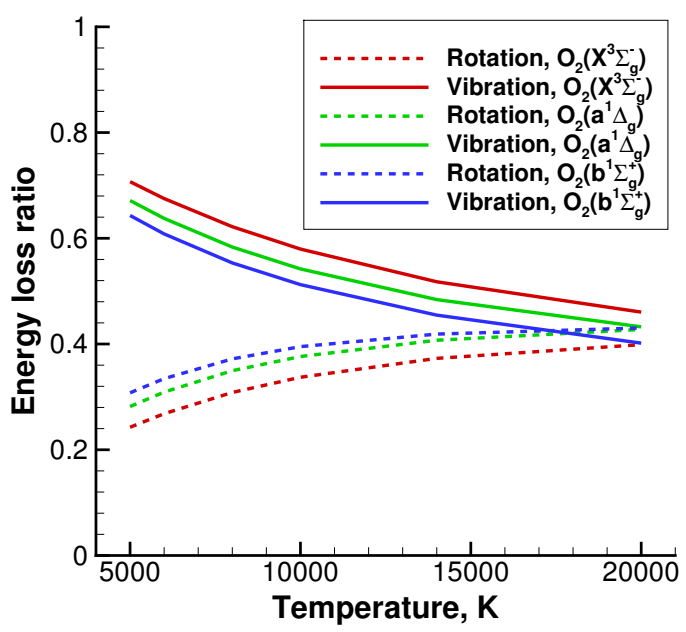

Figure 11. Rotational and vibrational energy losses due to dissociation of $\mathrm{O}_{2}\left(X^{3} \Sigma_{g}^{-}, a^{1} \Delta_{g}, b^{1} \Sigma_{g}^{+}\right)+\mathrm{Ar}$.

calculated by the quasi-classical trajectory method. The system of master equations constructed by the present state-to-state transition rates are solved to analyze the thermochemical nonequilibrium processes of $\mathrm{O}_{2}\left(X^{3} \Sigma_{g}^{-}, a^{1} \Delta_{g}, b^{1} \Sigma_{g}^{+}\right)+\mathrm{Ar}$ in various heat bath conditions.

For bound-bound transitions, the rotational temperature converges rapidly to the equilibrium temperature, and the rotational relaxation does not affect the vibrational relaxation at temperatures below $10,000 \mathrm{~K}$. However, at temperatures above $10,000 \mathrm{~K}$, the rotational nonequilibrium affects the vibrational relaxation through the rotational-to-vibrational energy transfer even though the rotational relaxation is much faster than that of vibration. In comparisons of the rotational and vibrational relaxations of the $X^{3} \Sigma_{g}^{-}, a^{1} \Delta_{g}$, and $b^{1} \Sigma_{g}^{+}$states, the rotational and vibrational relaxations of electronic ground $\mathrm{O}_{2}\left(X^{3} \Sigma_{g}^{-}\right)$are much slower than those of the electronically excited $\mathrm{O}_{2}\left(a^{1} \Delta_{g}, b^{1} \Sigma_{g}^{+}\right)$. These results show that the rotational and vibrational nonequilibrium of electronically excited $\mathrm{O}_{2}\left(a^{1} \Delta_{g}, b^{1} \Sigma_{g}^{+}\right)$must be treated as separate nonequilibrium processes in $\mathrm{O}_{2}+\mathrm{Ar}$ because, immediately behind a shock wave, the electronic excitation of $\mathrm{O}_{2}$ occurs readily through the heavy-particle impact processes.

For bound-free transitions, the rovibrational quasi-steady state (QSS) is clearly shown, and the nonequilibrium chemical reactions mainly occur in this QSS period. The nonequilibrium reaction rates of $\mathrm{O}_{2}\left(X^{3} \Sigma_{g}^{-}\right.$, $\left.a^{1} \Delta_{g}, b^{1} \Sigma_{g}^{+}\right)+$Ar are calculated with the QSS assumption in the present work. The dissociation reaction of $\mathrm{O}_{2}\left(X^{3} \Sigma_{g}^{-}\right)$is much slower than for the electronically excited $\mathrm{O}_{2}\left(a^{1} \Delta_{g}, b^{1} \Sigma_{g}^{+}\right)$. In comparisons of the reaction rates calculated from the rotational-vibrational-translational energy (RVT) and vibrational-translational energy (VT) transitions, it is shown that the reaction rates calculated by the RVT transitions are slightly slower. For the energy loss due to dissociation, it is observed that the normalized rotational and vibrational energy losses approach constant values of 0.45 , when the temperature increases.

\section{Acknowledgement}

The authors thank Dr. Jaffe, R. at NASA Ames Research Center and Dr. Park, C. at KAIST for valuable discussions about the oxygen chemistry and the evaluating of the potential energy surfaces. The authors also gratefully acknowledge funding for this work through Air Force Office of Scientific Research Grant FA9550-11-1-0309 and FA9550-12-1-0483.

\section{References}

\footnotetext{
${ }^{1}$ Steele, D. and Lippincott, E. R, "Comparative Study of Empirical Internuclear Potential Functions," Review of Modern Physics, Vol. 34, No 2, 1962, pp. 239-251.

${ }^{2}$ Vanderslice, J. T., Mason, E. A., Maisch, W. G., and Lippincott, E. R., "Ground State of Hydrogen by the Rydberg-
} 
Klein-Rees Method," Journal of Molecular Spectroscopy, Vol. 3, No. 1, 1959, pp. 17-29.

${ }^{3}$ Fujita, K. "Vibrational Relaxation and Dissociation Kinetics of CO by CO-O Collisions," AIAA 2008-3919.

${ }^{4}$ Kim, J. G. and Boyd, I. D., "Master Equation Analysis of Post Normal Shock Waves of Nitrogen," submitted in Journal of Thermophysics and Heat Transfers.

${ }^{5}$ Park. C, "Review of Chemical-Kinetic Problems of Future NASA Mission, I: Earth Entries," Journal of Thermophysics and Heat Transfer, Vol. 7, No. 3, 1993, pp. 385-398.

${ }^{6}$ Millikan, R. C. and White, D. R., "Systematics of Vibrational Relaxation," Journal of Chemical Physics, Vol. 39, No. 12, 1963, pp. 3209-3213.

${ }^{7}$ Gross, A. and Billing, G. D., "Rate Constants for Ozone Formation," Chemical Physics, Vol. 187, 1994, pp. 329-335.

${ }^{8}$ Gross, A. and Billing, G. D., "Isotope Effects on the Rate Constants for the Processes $\mathrm{O}_{2}+\mathrm{O} \rightarrow \mathrm{O}+\mathrm{O}_{2}$ and $\mathrm{O}_{2}+\mathrm{O}+\mathrm{Ar} \rightarrow \mathrm{O}_{3}+\mathrm{Ar}, "$ Chemical Physics, Vol. 217, 1997, pp. 1-18.

${ }^{9}$ Kroes, G. J. and Rettschnick, R. P. H., "Vibrational Relaxation of Glyoxal in Collisions with He and Ar," Chemical Physics, Vol. 156, 1991, pp. 293-307.

${ }^{10}$ Eaker, C. W., "A Fast Fourier Transform Method for Quasiclassical Selection of Initial Coordinates and Momenta for Rotating Diatoms," Journal of Chemical Physics, Vol. 90, No. 1, 1989, pp. 105-111.

${ }^{11}$ Miller, W. H. (ed), Dynamics of Molecular Collisions, Plenum, New York, 1976.

${ }^{12}$ Bernstein, r. B. (ed.) Atom-Molecular Collision Theory: A Guide for the Experimentalist, Plenum, New York, 1979.

${ }^{13}$ Camac, M., "O $\mathrm{O}_{2}$ Vibration Relaxation in Oxygen-Argon Mixtures," Journal of Chemical Physics, Vol. 34, No. 2, 1961, pp. $448-459$.

${ }^{14}$ Landau, L. and Teller, E., "Theory of Sound Dispersion," Physics Sowjetunion, Vol. 10, 1936, pp. 34-43.

${ }^{15}$ Park, C., "Rotational Relaxation of $\mathrm{N}_{2}$ Behind a Strong Shock Wave," Journal of Thermophysics and Heat Transfer, Vol. 18, No. 4, 2004, pp. 527-533.

${ }^{16}$ Park, C., Nonequilibrium Hypersonic Aerothermodynamics, Wiley, New york, 1990.

${ }^{17}$ Kim, J. G. and Boyd, I. D., "State-Resolved Master Equation Analysis of Thermochemical Nonequilibrium of Nitrogen," Chemical Physics, Vol. 415, 2013, pp. 237-246.

${ }^{18}$ Baulch, D. L., Drysdale, D. D., and Horne, D. G., Evaluated Kinetic Data for High Temperature Reactions, Vol. 2, CRC Press, London, 1973. 